\title{
Taking Advantage of Overhearing in Low Power Listening WSNs: A Performance Analysis of the LWT-MAC Protocol
}

\author{
Cristina Cano • Boris Bellalta • Anna Sfairopoulou • \\ Miquel Oliver · Jaume Barceló
}

(C) Springer Science+Business Media, LLC 2010

\begin{abstract}
LWT-MAC is a new Low Power Listening MAC protocol for WSNs designed to rapidly react to instantaneous increases of the network load. It takes advantage of overhearing by waking up all nodes at the end of a transmission to send or receive packets without needing to transmit the long preamble before. In this work, detailed analytical models of the LWT-MAC and B-MAC protocols, for both saturated and unsaturated conditions, are presented. Moreover, the key LWT-MAC parameters are optimized in order to minimize the energy consumption, constrained to obtain the same throughput as the IEEE 802.11 (CSMA/CA) MAC protocol. From the behavior of the optimal LWT-MAC parameters, a heuristic configuration is proposed. Finally, the LWT-MAC is compared to BMAC, in both single and multi-hop scenarios, showing improvements in energy consumption, throughput and delay.
\end{abstract}

Keywords WSNs • low power listening - B-MAC • LWT-MAC $\cdot$ analytical model

\section{Introduction}

Wireless Sensor Networks (WSNs) consist of small devices that sense environmental data and send it to

C. Cano $(\bowtie) \cdot$ B. Bellalta $\cdot$ A. Sfairopoulou

M. Oliver · J. Barceló

NeTS Research Group,

Department of Information and Communication

Technologies, Universitat Pompeu Fabra, C/ Tanger,

122-140, 08018, Barcelona, Spain

e-mail: cristina.cano@upf.edu a central collector. Normally, sensor nodes have reduced processing, memory and battery resources and are deployed in remote and large areas. The battery replacement in these networks is, therefore, too costly or even impossible, making energy consumption the most important constraint. For this reason, the Medium Access Control (MAC) protocol is crucial as it directly influences the transceiver operation that is the most consuming component of a sensor node. The common approach to reduce energy consumption in WSNs is to periodically put the transceiver into sleep mode, working in a low duty cycle operation instead of continuously listening the channel as in traditional wired and wireless networks without power constraints, like in IEEE 802.11 Carrier Sense Multiple Access with Collision Avoidance (CSMA/CA) [1].

A well-known MAC protocol for WSNs is Berkeley MAC (B-MAC) [2] in which each node periodically and independently of the others samples the radio channel to detect activity, what is known as Low Power Listening (LPL) operation. Then, when a node wants to send a message, it first sends a preamble long enough to overlap with the listening time (active part of the duty cycle) of the receiver. Using B-MAC the energy consumption of the sensor nodes is extremely reduced at very low loads. However, as the load increases, for instance, due to events occurrence, the collisions of preambles become a significant energy waste, even more important in large scale WSNs with hidden terminal problems.

The Low power listening with Wake up after Transmissions MAC (LWT-MAC) protocol (presented by the authors in [3]) was designed to maintain a low energy consumption at low loads while, at the same time, being able to react to instantaneous increases of the 
network load. A local synchronization after transmissions, that ensures that all nodes that have overheard the last transmission will be awake to receive a new message, is adopted, hence without requiring the long preamble transmission.

Analytical models of WSNs MAC protocols allow to derive performance optimizations of the different parameters involved: duty cycle, Contention Window (CW) or packet size among others, depending on different network scenarios. However, existing analytical models of WSNs MAC protocols (for instance, the ones used in $[2,4])$ are extremely simple as they only consider the time to transmit a packet without analyzing the time and energy wasted in collisions. Although these simple analytical models are valid for low traffic loads they become inaccurate when the traffic load increases. More detailed analytical models of scheduled MAC protocols such as the Sensor-MAC (S-MAC) [5] and nanoMAC [6] have been presented, however, the LPL operation has not been studied so exhaustively.

In this work, the LWT-MAC protocol is studied from an analytical point of view. The presented LWT-MAC analytical model considers the energy waste due to collisions and overhearing in a single-hop network under saturated and unsaturated conditions. The analytical model presented is adapted to model the B-MAC protocol and it can also be extended to model other LPL MAC protocols (like the X-MAC protocol [7]). Using the analytical model, the LWT-MAC key network parameters are identified and optimized to minimize the energy consumption but maintaining a good performance in terms of delay and throughput. From the optimization process, a heuristic configuration is derived. The LWT-MAC with the heuristic configuration is compared with B-MAC in a single-hop network and in a multi-hop scenario with periodic and event-based traffic profiles.

The rest of the paper is organized as follows: Section 2 provides a comparison of the proposed approach with similar existing mechanisms, then, in Section 3 the LWT-MAC is described. The LWT-MAC analytical model and the adaptation to model the BMAC protocol, as well as their validation are presented in Section 4. The optimization analysis of the LWTMAC protocol is performed in Section 5 while the performance results are discussed in Section 6. Finally, some concluding remarks are given in Section 7.

\section{Related work}

LPL MAC protocols as B-MAC [2] perform well when the traffic load of the network is low, however as the traffic load increases the continuous collisions of preambles considerably decreases the network performance. The LWT-MAC protocol aims at improving the performance by waking up neighboring nodes at the end of a transmission in order to send or receive packets [3].

Some similar mechanisms have already been defined in order to address the same problem. For instance, the multi-hop streaming capability defined in the Scheduled Channel Polling MAC (SCP-MAC) protocol [8] that increases the duty cycle upon a message reception in order to reduce the end-to-end delay. Another example is described in [9] where the sender of a message activates a send more bit in the header of the packet indicating that there is more data pending to be transmitted. The destination of the message stays awake at the end of the transmission to receive the packet, thus eliminating the need of the long preamble transmission. In scheduled MAC protocols the same idea can be followed: in S-MAC [4] and Timeout-MAC (T-MAC) [10] each node that overhears an Request To Send (RTS) or Clear to Send (CTS) stays awake just in case it should forward the message.

All those mechanisms differ from the approach presented in this work in the sense that in LWT-MAC every node that has overheard a transmission is allowed to transmit at the end, not only the sender or the recipient of the last transmission. This approach improves the performance when the traffic load of the network increases. The load of the network can increase, for instance, due to an event detection, in this situation a set of nearby nodes try to transmit a packet to inform the sink about the event occurrence.

Other approaches like the X-MAC protocol [7] aim at estimating the traffic load of the network and then adapt the duty cycle accordingly. Those protocols provide better performance but they suffer from an increased complexity. The LWT-MAC protocol, in contrast, adapts to the traffic load in a simple manner, only by waking up all neighboring nodes after a transmission.

\section{Taking advantage of overhearing: the LWT-MAC protocol}

The LWT-MAC extends the operation of B-MAC by taking advantage of the local synchronization of all nodes that overhear a transmission [3]. The LWT-MAC defines that all overhearing nodes wake up simultaneously at the end of each successful transmission in order to send or receive packets. Since all nodes that have overheard the last transmission will be awake, the long 


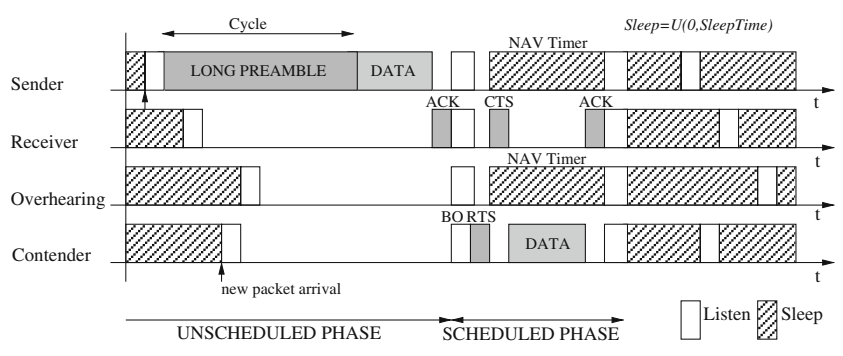

(a) LWT-MAC Protocol

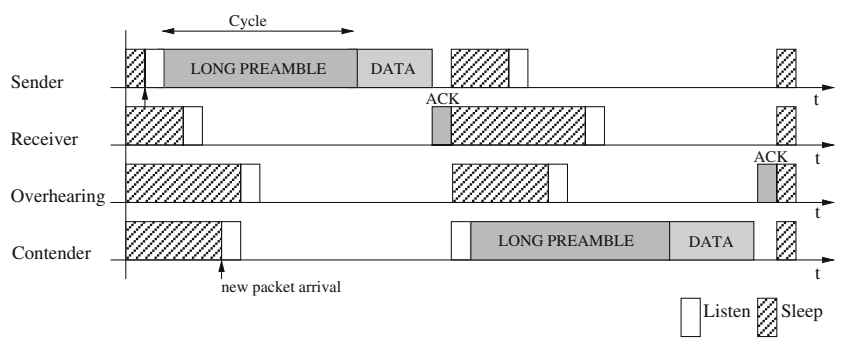

(b) B-MAC Protocol

Fig. 1 Comparison of LWT-MAC and B-MAC medium access mechanisms

preamble is no longer necessary (the medium access mechanism is depicted in Fig. 1a).

In this scheduled phase it is mandatory to compute a random backoff $(\mathrm{BO})$ before attempting transmission, however in the unscheduled phase it is optional. This forces all nodes to listen after each transmission at least the value of the $\mathrm{CW}$. During the random BO of the scheduled phase nodes should keep listening to the channel in case any other node starts a transmission.

The use of the RTS/CTS transaction before the packet transmission in the scheduled phase is recommended. The four-way handshake will help in alleviating hidden terminal problems and it is useful to let overhearing nodes sleep during the entire packet transmission. The duration of the transmission should be included in RTS, CTS and Data messages and will allow the overhearing nodes to set a Network Allocation Vector (NAV) timer (as defined in [1]) and sleep until the transmission finishes.

If no message is sent during the listening after transmissions period, nodes go to sleep for a random time (with a maximum value equal to the sleep time of the duty cycle) moving towards the unscheduled phase.

Observe that, compared to B-MAC (Fig. 1b), there is a reduction of the energy waste, both in transmission and reception, due to the suppression of continuous long preamble transmissions. Moreover, by suppressing long preambles the delay and the channel occupation durations are reduced as well. Additionally, at instantaneous increases of the network load the protocol reduces the time required to send a packet improving the network performance. However, it should be noted that the performance benefits of LWT-MAC mainly depend on the probability that the next transmission receiver has overheard the last transmission, that in turn depends on the topology and on how the routes to the sink are selected.

If a packet transmission fails, a retransmission procedure is initiated. The details of this procedure are different in the scheduled and unscheduled modes of operation.

\subsection{Retransmission procedure in the unscheduled access}

If a transmission failure occurs during the unscheduled access, the acknowledgement (ACK) is not received, the sender retransmits the packet by sending an RTS after waiting a random $\mathrm{BO}$. As the transmission failure can be caused by collisions of preambles, the retransmission increases the probability to receive the RTS correctly. After that, in case the CTS is not received (there are two consecutive transmission failures), it is assumed that the intended recipient is either involved in another transmission or waiting for a transmission to finish. In order to alleviate consecutive collisions, the sender does not immediately retransmit the message. Instead, it waits a Collision Avoidance (CA) timer (set to the duration of a preamble and a frame transmission). During the CA timer the node keeps listening to the channel, that provides the opportunity to get synchronized with the current ongoing transmission (if any) after overhearing a message involved. If that is the case, it can sleep until the transmission finishes and retry transmission using the scheduled method. Otherwise, if the CA timer expires, the node retries transmission using the long preamble again. The CA timer has been added as an optional mechanism to reduce hidden terminal problems, however, it can be deactivated when needed, for instance in single-hop networks in which all nodes are inside the coverage range of the others.

The RTS/CTS mechanism can also be activated in the unscheduled mode [2], i.e., an RTS is sent after the transmission of the long preamble. In this case, if a transmission failure occurs (CTS not received) the sender assumes that the recipient is involved in another transmission and waits the CA timer as previously described. Note that, after a collision among hidden terminal nodes, the receiver will hopefully receive at least one of the RTS sent. By activating the RTS/CTS in the unscheduled mode the use of the NAV timer 
will also allow to let overhearing nodes to sleep as previously explained.

\subsection{Retransmission procedure in the scheduled access}

If a transmission fails during the scheduled access it can be either because the CTS or the ACK are not received. In case the RTS fails (CTS not received), it is assumed that the recipient has not overheard the past transmission and, therefore, it is sleeping (notice that the long preamble has not been previously sent). In this case, the node will retry to send the message by sending the long preamble first in order to wake up the receiver. If the packet transmission is not acknowledged the sender waits a CA timer as already explained in the unscheduled access.

The detailed specification of the protocol behavior is depicted in Fig. 2.

\subsection{Performance benefits}

For illustration purposes the energy consumption and throughput of LWT-MAC compared with the results obtained using IEEE 802.11 [1] and B-MAC in a 10node single hop network are shown in Figs. 3 and 4 (the parameters used are depicted in Table 1). Observe that the throughput is significantly improved if compared to B-MAC at the cost of a slightly higher energy consumption at low loads, where idle listening after transmission periods occur. However, the energy consumption is reduced at high loads due to the suppression of the long preamble transmission.

\subsection{Addressing collective Quality of Service (QoS)}

The LWT-MAC wake up after transmissions capability makes it a good candidate to be used in event-based

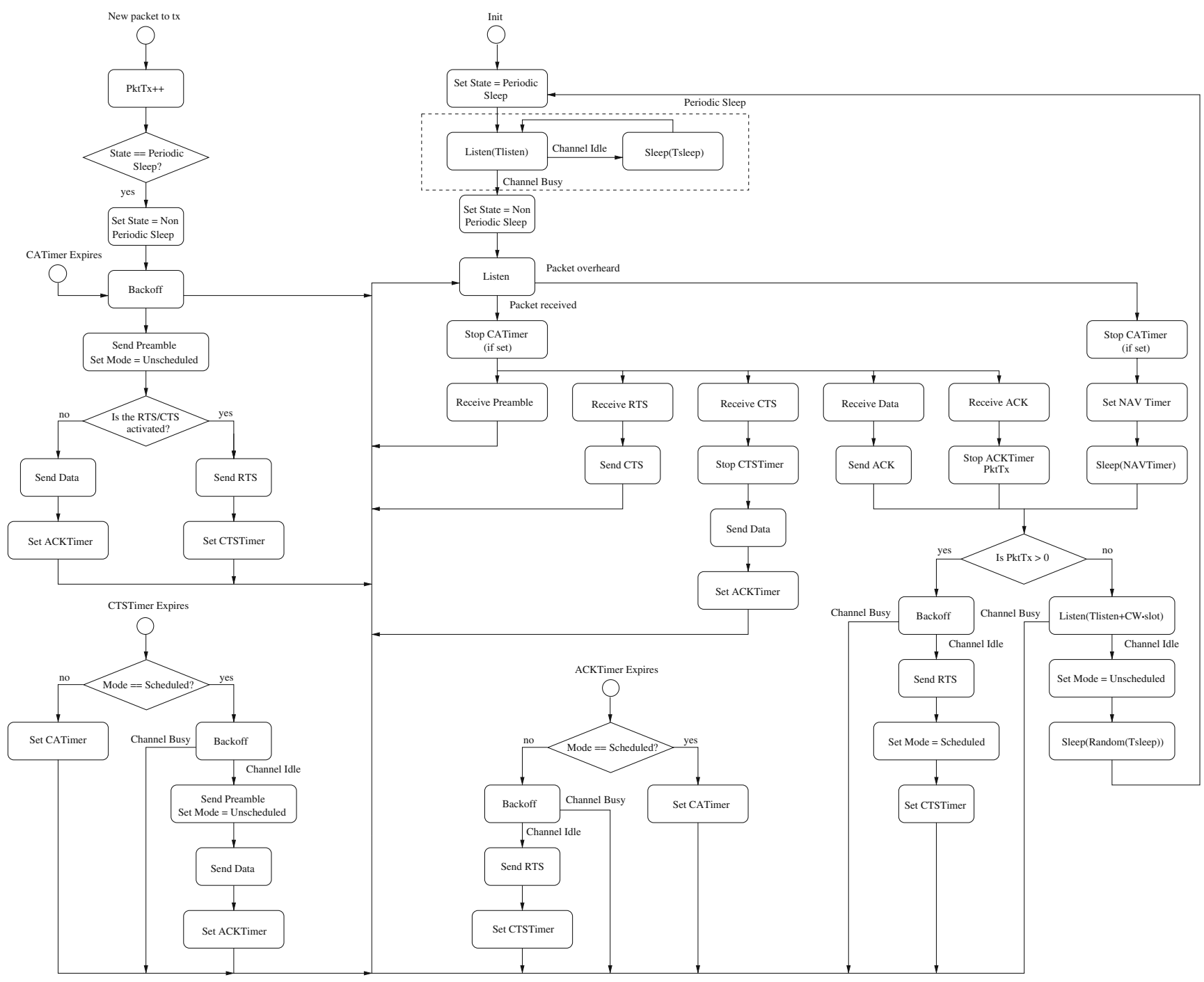

Fig. 2 Flowchart of the LWT-MAC protocol. The Mode value is initialized to Unscheduled and PktTx is initialized to zero 


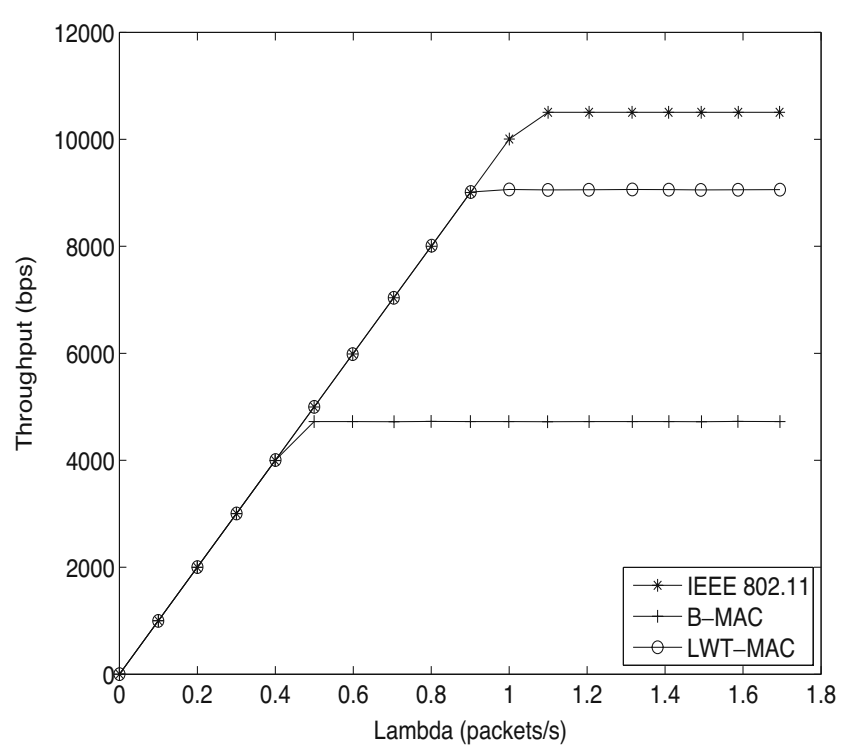

Fig. 3 Throughput of IEEE 802.11, B-MAC and LWT-MAC in a 10-node single hop network

WSNs where instantaneous increases of the traffic load occur due to event detection at nearby sensor nodes. The QoS observed by event-based messages is crucial in order to assure the correct and fast event detection at sink. However, this QoS differs from the traditional definition in which the QoS measurement is made packet by packet. In event-based WSNs the QoS should refer to the group of messages related to an event, it is known as collective QoS. Collective QoS is defined as the QoS (delay, bandwidth, packet loss, etc.) of the set of packets related to a specific event [11]; i.e., the delay

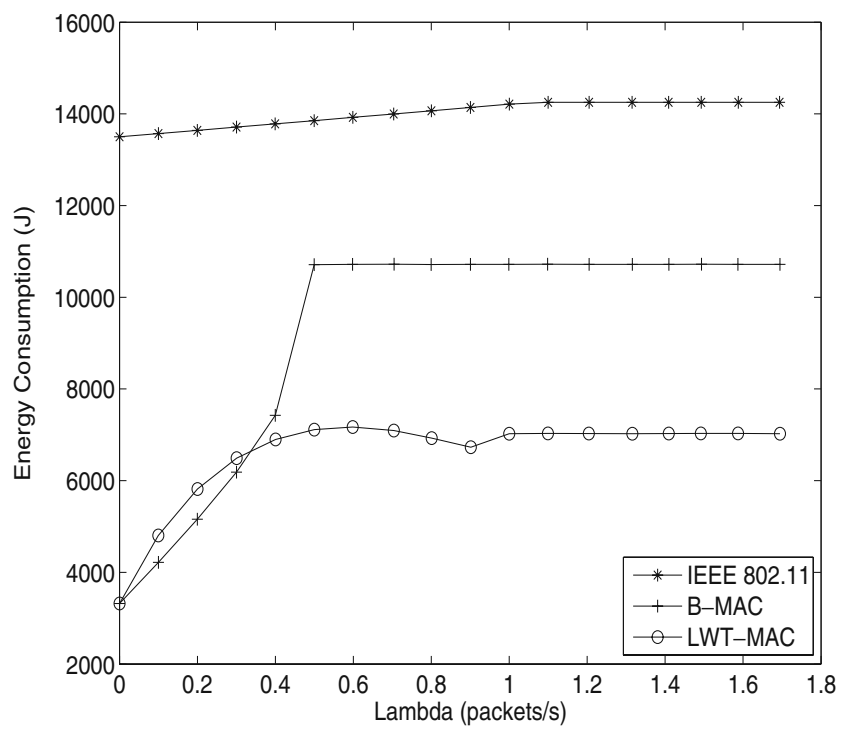

Fig. 4 Energy consumption of IEEE 802.11, B-MAC and LWTMAC in a 10-node single hop network
Table 1 Default parameters

\begin{tabular}{llll}
\hline Parameter & Value & Parameter & Value \\
\hline$r$ (data rate) & $20 \mathrm{kbps}$ & $T_{\text {listen }} / T_{\text {sleep }}$ & $24.5 / 75.5 \mathrm{~ms}$ \\
$\sigma$ (empty slot) & $1 \mathrm{~ms}$ & $L_{\text {data }}($ packet size $)$ & $1,000 \mathrm{bits}$ \\
DIFS & $10 \mathrm{~ms}$ & $L_{\mathrm{rts}}, L_{\text {cts }}, L_{\text {ack }}$ & $64 \mathrm{bits}$ \\
SIFS & $5 \mathrm{~ms}$ & $E_{\mathrm{tx}}$ & $24.75 \mathrm{~mW}$ \\
$\mathrm{CW}$ & 64 & $E_{\mathrm{rx}}, E_{\text {idle }}$ & $13.5 \mathrm{~mW}$ \\
$K$ (queue size) & $100 \mathrm{pkts}$ & $E_{\text {sleep }}$ & $0.015 \mathrm{~mW}$ \\
$R$ (retry limit) & 7 & $T$ (time) & $1 \cdot 10^{5} \mathrm{~s}$ \\
\hline
\end{tabular}

of the individual messages is not crucial but the latency from the event generation until the event detection at sink is critical. A MAC protocol that efficiently reacts to event-based traffic will increase the collective QoS of the messages involved.

The LWT-MAC protocol is designed in order to improve the collective QoS of event messages while maintaining a low energy consumption. As far as the authors know, there is not any other MAC protocol designed keeping in mind collective QoS metrics. Those which are focused on QoS are based on end-to-end traditional QoS metrics instead [12-17].

The behavior of the protocol with periodic and event-based traffic profiles and its ability to increase the collective QoS was studied in [18].

\section{LWT-MAC analytical model}

In this section an analytical model of the LWT-MAC protocol in single-hop WSNs is described. The analytical model assumes ideal channel conditions (no channel errors or hidden terminal problems) and that each node computes a random $\mathrm{BO}$ (between 0 and $\mathrm{CW}$ ) before each transmission attempt. ${ }^{1}$ For simplicity reasons the sensor nodes are considered to be homogeneous (equal traffic profiles and capabilities). Table 2 provides the description of some relevant variables used.

The extension of the analytical model to a multi-hop network is a challenging task since collisions can happen due to hidden terminal problems [19]. Moreover, in WSNs, where hidden terminals can wake up at any moment during an ongoing transmission, the multi-hop analysis becomes even more difficult than in traditional wireless networks where nodes are always listening to the channel. Therefore, the multi-hop analysis is left for future study.

\footnotetext{
${ }^{1}$ The effect of the CA timer previously described has not been studied, it is considered that collisions move the system to the unscheduled mode.
} 
Table 2 Notation

\begin{tabular}{ll}
\hline Notation & Description \\
\hline$n$ & Number of nodes \\
$L_{\text {data }}$ & Packet size (bits) \\
$\lambda$ & Rate of packet generation (packets/s) \\
$K$ & Queue size (packets) \\
$L_{\mathrm{rts}, \mathrm{cts}, \text { ack }}$ & RTS,CTS,ACK packet size (bits) \\
$L_{\mathrm{p}}$ & Preamble size (bits) \\
$r$ & Transmission rate (bits/s) \\
$T$ & Time (s) \\
$S$ & Throughput (bits/s) \\
$M$ & Average number of transmission attempts per packet \\
$B$ & Average number of slots in BO \\
$p_{\text {sch }}$ & Scheduled probability \\
$p_{\mathrm{w}}$ & Probability to wake up after a transmission \\
$\rho$ & Queue utilization \\
$\tau$ & Transmission probability in a given slot \\
$\mathrm{CW}$ & Contention window of the random BO \\
$\sigma$ & Empty slot duration (s) \\
$\zeta$ & Refers to the metrics: $S, M, B, p_{\text {sch }}, p_{\mathrm{e}}, \rho$ \\
$T_{\text {sleep }}$ & Sleep time of the duty cycle (s) \\
$T_{\text {listen }}$ & Listen time of the duty cycle (s) \\
\hline &
\end{tabular}

\subsection{Network performance metrics}

The LWT-MAC analytical model is based on the one described in [20] where an IEEE 802.11 (CSMA/CA) analytical model to compute traditional metrics such as throughput, delay and queue occupation is presented.

A sensor node is modeled as a single queue of length $K$ packets. Each node generates packets following a Poisson distribution with rate $\lambda$ packets/s and average packet length $L_{\text {data }}$ bits. From these assumptions the following metrics can be computed:

$A=\lambda X, \quad \rho=A\left(1-P_{\mathrm{b}}\right), \quad P_{\mathrm{b}}=\frac{(1-A) A^{K}}{1-A^{K+1}}$

where $A$ is the offered load, $\rho$ is the queue utilization, $P_{\mathrm{b}}$ denotes the blocking probability and $X$ refers to the service time (the time since the packet arrives at the head of the queue until it is released from it, assuming that it follows an exponential distribution).

The service time can be calculated as:

$X=(M-1)\left(B \alpha+T_{\mathrm{c}}\right)+B \alpha+T_{\mathrm{s}}$

where $M$ is the average number of required transmission attempts per packet, $B$ is defined as the average number of slots selected before each transmission attempt, $\alpha$ is the average slot duration and $T_{\mathrm{c}}$ and $T_{\mathrm{s}}$ are the durations of a collision and a successful transmission respectively.
The average number of attempts per packet successfully transmitted or discarded $(M)$ due to maximum retry limit $(R)$ reached is computed as:

$M=\frac{1-p^{R+1}}{1-p}$

where $p$ is the conditional collision probability (assumed to be constant for all transmission attempts):

$p=1-(1-\tau)^{n-1}$

Being $n$ the total number of nodes in the network and $\tau$ the steady state probability that a node transmits in a random slot given that it has a packet ready to be transmitted:

$\tau=\frac{\rho}{B+1}$

Assuming that the $\mathrm{BO}$ is uniformly distributed in the range [0-CW], $B$ can be obtained as shown in Eq. 6 .

$B=\frac{\mathrm{CW}-1}{2}$

The average slot duration $(\alpha)$, is calculated considering the duration of the slot depending on the channel state (Eq. 7). As the channel is assumed to be errorfree, it can only be in empty, successful or collision states with their corresponding probabilities $p_{\mathrm{e}}, p_{\mathrm{s}}, p_{\mathrm{c}}$.

$\alpha=p_{\mathrm{e}} \sigma+p_{\mathrm{s}}\left(T_{\mathrm{s}}+\sigma\right)+p_{\mathrm{c}}\left(T_{\mathrm{c}}+\sigma\right)$

where $\sigma$ is the empty slot duration.

The channel state probabilities are related to the stationary probability that the rest of the nodes (except the one that is in $\mathrm{BO}$ ) try to transmit in a given random slot. These are given by:

$$
\begin{aligned}
& p_{\mathrm{e}}=(1-\tau)^{n-1} \\
& p_{\mathrm{s}}=(n-1) \tau(1-\tau)^{n-2} \\
& p_{\mathrm{c}}=1-p_{\mathrm{e}}-p_{\mathrm{s}}
\end{aligned}
$$

Finally, the throughput per node can be computed as:

$S=\rho \frac{L_{\text {data }}}{X}\left(1-p_{\mathrm{d}}\right)$

where $p_{\mathrm{d}}$ is the probability to discard a packet due to maximum retry limit reached:

$p_{\mathrm{d}}=p^{R+1}$

The channel occupation durations (Eqs. 11 and 12) can be computed considering the length of the messages involved and the time intervals between them. Note that in the unscheduled mode the preamble is sent before each packet transmission attempt. The analytical 
model presented in this work only considers the transmission of packets preceded by RTS/CTS messages, although the model is easily adaptable to also consider the basic access method. Observe also that it has been considered the use of the DIFS, SIFS and EIFS time periods as in the IEEE 802.11 (CSMA/CA) [1].

$T_{\mathrm{c}}=\mathrm{DIFS}+\frac{\left(L_{\mathrm{p}} \cdot p_{\mathrm{unsch}}\right)+L_{\mathrm{rts}}}{r}+\mathrm{EIFS}$

$T_{\mathrm{s}}=\mathrm{DIFS}+\frac{\left(L_{\mathrm{p}} \cdot p_{\mathrm{unsch}}\right)+L_{\mathrm{rts}}+L_{\mathrm{cts}}+L_{\mathrm{data}}+L_{\mathrm{ack}}}{r}$

$$
+3 \text { SIFS }
$$

where $L_{\mathrm{p}}, L_{\mathrm{rts}}, L_{\mathrm{cts}}$ and $L_{\mathrm{ack}}$ are the lengths of the preamble, RTS, CTS and ACK messages respectively, while $r$ refers to the transmission rate. The probability of transmitting a packet in scheduled mode $\left(p_{\text {sch }}\right)$ is the probability that after a successful transmission, any other node has still data to be transmitted. On the other hand, the probability to transmit a packet in the unscheduled mode ( $p_{\text {unsch }}$ ) is obtained as the complementary of the former:

$p_{\text {sch }}=\frac{p_{\text {ss }}}{1-p_{\text {es }}}\left(1-(1-\rho)^{n}\right), \quad p_{\text {unsch }}=1-p_{\text {sch }}$

where $p_{\text {ss }}$ and $p_{\text {es }}$ are the probabilities of successful and empty slots from the network point of view:

$p_{\mathrm{es}}=(1-\tau)^{n}, \quad p_{\mathrm{ss}}=n \tau(1-\tau)^{n-1}$

The analytical model is solved using a fixed point approximation. With the metrics obtained the energy consumption of a sensor node during a certain amount of time can be computed.

\subsection{Energy consumption}

The total energy consumption of a sensor node can be divided in four parts: (i) the energy spent to transmit and (ii) receive messages, (iii) the energy wasted in overhearing, and (iv) the energy spent in duty cycle (sleeping and waking up in inactive periods):

$e=e_{\mathrm{tx}}+e_{\mathrm{rx}}+e_{\mathrm{ov}}+e_{\mathrm{dc}}$

Let $N_{\mathrm{s}}$ be the total number of messages a node successfully sends during a time $T$, it can be derived using the throughput $S$ and the packet length $L_{\text {data }}$ as $N_{\mathrm{s}}=T\left(\frac{S}{L_{\mathrm{data}}}\right)$.

The energy spent to transmit $N_{\mathrm{s}}$ messages is computed taking into account the energy needed to successfully transmit a packet $\left(e_{\mathrm{s}, \mathrm{tx}}\right)$ and the energy spent in collisions for each unsuccessful attempt $\left(e_{\mathrm{c}, \mathrm{tx}}\right)$ :

$e_{\mathrm{tx}}=N_{\mathrm{s}}\left(e_{\mathrm{s}, \mathrm{tx}}+(M-1) e_{\mathrm{c}, \mathrm{tx}}\right)$
The values of $e_{\mathrm{s}, \text { tx }}$ and $e_{\mathrm{c}, \text { tx }}$ can be obtained considering the energy spent to transmit and receive the messages involved and the empty time intervals (Eqs. 17 and 18). Moreover, the empty slots of the BO procedure must be considered (note that the busy slots of the BO countdown are part of the receiving or overhearing energy consumptions).

$$
\begin{aligned}
e_{\mathrm{c}, \mathrm{tx}}= & E_{\mathrm{idle}}\left(\mathrm{DIFS}+B \sigma p_{\mathrm{e}}+2 \operatorname{SIFS}+\frac{L_{\mathrm{cts}}}{r}\right) \\
& +E_{\mathrm{tx}} \frac{\left(L_{\mathrm{p}} \cdot p_{\mathrm{unsch}}\right)+L_{\mathrm{rts}}}{r}
\end{aligned}
$$

$$
\begin{aligned}
e_{\mathrm{s}, \mathrm{tx}}= & E_{\mathrm{idle}}\left(\mathrm{DIFS}+B \sigma p_{\mathrm{e}}+3 \mathrm{SIFS}+T_{\mathrm{e}}\right) \\
& +E_{\mathrm{tx}} \frac{\left(L_{\mathrm{p}} \cdot p_{\mathrm{unsch}}\right)+L_{\mathrm{rts}}+L_{\mathrm{data}}}{r} \\
& +E_{\mathrm{rx}} \frac{L_{\mathrm{cts}}+L_{\mathrm{ack}}}{r}
\end{aligned}
$$

where $E_{\mathrm{tx}}, E_{\mathrm{rx}}$ and $E_{\text {idle }}$ denote the energy consumptions of being in transmission, reception and idle modes.

Notice that, after a successful transmission, all nodes in the network keep listening to the channel in case another node has something to transmit. If there are no more data to send, this time remains empty and each node stays in the idle mode during a $T_{\text {listen }}$ (added to avoid synchronization problems) plus the $\mathrm{CW}$, this is:

$T_{\mathrm{e}}=(1-\rho)^{n}\left(T_{\text {listen }}+\sigma \mathrm{CW}\right)$

For simplicity reasons, it has been considered that each node receives $N_{\mathrm{s}}$ packet destined to it. Therefore, the total energy consumption to receive those messages is computed as:

$e_{\mathrm{rx}}=N_{\mathrm{s}} \cdot e_{\mathrm{s}, \mathrm{rx}}$

The colliding packets have not been considered here. It is assumed that the unsuccessful transmissions of a node collide with those that are destined to it and that the probability to collide with more than one packet can be neglected.

The energy consumption to receive a packet is:

$$
\begin{aligned}
e_{\mathrm{s}, \mathrm{rx}}= & p_{\mathrm{sch}} \cdot e_{\mathrm{b}}+p_{\mathrm{unsch}} \cdot e_{\mathrm{p}}+E_{\mathrm{idle}}\left(3 \mathrm{SIFS}+T_{\mathrm{e}}\right) \\
& +E_{\mathrm{rx}}\left(\frac{L_{\mathrm{rts}}+L_{\mathrm{data}}}{r}\right)+E_{\mathrm{tx}}\left(\frac{L_{\mathrm{cts}}+L_{\mathrm{ack}}}{r}\right)
\end{aligned}
$$

where $e_{\mathrm{b}}$ is the energy of a busy listening after transmissions period, i.e., the idle time interval before sending a packet in the scheduled mode. Observe that, those nodes without data to send must also wait the remaining $\mathrm{BO}$ of the other nodes. 
This time has been approximated by $B \sigma$ as shown in Eq. 22. This approximation does not affect when the traffic load is low (the scheduled probability is small) or high (the probability that a node has no data to transmit is negligible). However, it affects the results with moderate traffic load and its effect depends on the number of nodes. When $n$ is high the energy consumption is overestimated (as the probability that a node has a small remaining BO increases) while with small $n$ the energy consumed is underestimated (since the probability to transmit a packet that arrives at the queue during the listen after transmission period increases). Then:

$e_{\mathrm{b}} \approx E_{\mathrm{idle}}(\mathrm{DIFS}+B \sigma(1-\rho))$

The parameter $e_{\mathrm{p}}$ refers to the energy spent receiving the long preamble. If a node has something to transmit it will be listening to the channel, therefore it will receive the entire long preamble of any other transmission in the medium. Otherwise, the node will be in duty cycle mode and on average it will wake up in the middle of the other's long preamble transmissions plus its own $T_{\text {listen }}$ :

$$
\begin{aligned}
e_{\mathrm{p}}= & \rho\left(E_{\text {idle }} \mathrm{DIFS}+E_{\mathrm{rx}} \frac{L_{\mathrm{p}}}{r}\right) \\
& +(1-\rho)\left(\frac{E_{\text {idle }} T_{\text {listen }}+E_{\mathrm{rx}} \frac{L_{\mathrm{p}}}{r}}{2}\right)
\end{aligned}
$$

Similarly, the energy consumption due to overhearing is computed as:

$e_{\mathrm{ov}}=N_{\mathrm{s}}(n-2)\left(e_{\mathrm{s}, \mathrm{ov}}+\frac{M-1}{2} e_{\mathrm{c}, \mathrm{ov}}\right)$

where $e_{\mathrm{s}, \text { ov }}$ and $e_{\mathrm{c}, \text { ov }}$ are the energy consumption to overhear a successful transmission or a collision respectively:

$$
\begin{aligned}
e_{\mathrm{c}, \mathrm{ov}}= & p_{\mathrm{sch}} \cdot e_{\mathrm{b}}+p_{\mathrm{unsch}} \cdot e_{\mathrm{p}}+E_{\mathrm{rx}} \frac{L_{\mathrm{rts}}}{r} \\
& +E_{\mathrm{idle}}\left(2 \operatorname{SIFS}+\frac{L_{\mathrm{cts}}}{r}\right) \\
e_{\mathrm{s}, \mathrm{ov}}= & p_{\mathrm{sch}} \cdot e_{\mathrm{b}}+p_{\text {unsch }} \cdot e_{\mathrm{p}}+E_{\mathrm{idle}} T_{\mathrm{e}}+E_{\mathrm{rx}} \frac{L_{\mathrm{rts}}}{r} \\
& +E_{\text {sleep }}\left(\frac{L_{\mathrm{cts}}+L_{\mathrm{data}}+L_{\mathrm{ack}}}{r}+3 \mathrm{SIFS}\right)
\end{aligned}
$$

where $E_{\text {sleep }}$ denotes the energy of being in sleep mode.

Finally, the rest of time ( $\left.T_{\text {inactive }}\right)$, that can be obtained using the equations above computing the time instead of the energy, and the total time $T$, each node performs a low duty cycle operation, listening and sleeping according to the duty cycle:

$e_{\mathrm{dc}}=T_{\text {inactive }}\left(E_{\text {idle }} \frac{T_{\text {listen }}}{T_{\mathrm{ci}}}+E_{\text {sleep }} \frac{T_{\text {sleep }}}{T_{\mathrm{ci}}}\right)$

where $T_{\mathrm{ci}}$ is the check interval:

$T_{\text {ci }}=T_{\text {listen }}+T_{\text {sleep }}$

\subsection{B-MAC analytical model}

The previous analytical model can be adapted to model the behaviour of the B-MAC protocol. To achieve that aim two modifications are needed: (i) the probability of being in scheduled mode $\left(p_{\text {sch }}\right)$ has to be fixed to 0 , meaning that the protocol always works in the unscheduled mode and (ii) the empty listen time after a successful transmission has not to be considered $\left(T_{\mathrm{e}}=\right.$ 0 ) when computing the energy consumption of a sensor node since the listen after transmissions capability does not apply in B-MAC.

\subsection{Analytical model validation}

The SENSE simulator [21] has been used to validate the results obtained with the analytical model. The scenario consists of $n$ nodes randomly placed in an area smaller than the maximum coverage range, thus, assuming ideal channel conditions, a full connectivity among them is assured. The default parameters used for the evaluation are shown in Table 1 (see Section 3).

Figure 5 shows the analytical and simulation results of B-MAC and LWT-MAC with different number of sensor nodes. Observe how the scheduled probability of LWT-MAC (Fig. 5a) increases with the traffic load until a certain point at which the number of collisions (that move the system to the unscheduled mode) is noticeable. With a higher number of nodes, the scheduled probability increases faster (since the traffic load is higher), however it also becomes constant sooner as the collision probability is also higher. The total energy consumption of LWT-MAC (Fig. 5b) is strongly related to the scheduled probability. Note how the model underestimates the energy consumption approaching saturation for two and five nodes and overestimates it with ten nodes. The reason for this discrepancy is the aforementioned approximation on the value of $e_{\mathrm{b}}$. In contrast, for B-MAC, since it always works in unscheduled mode, its accuracy is not affected by the $e_{\mathrm{b}}$ approximation. It is also interesting to observe the throughput (Fig. 5c) that slightly increases, in both protocols, with the number of nodes, however the saturation through- 


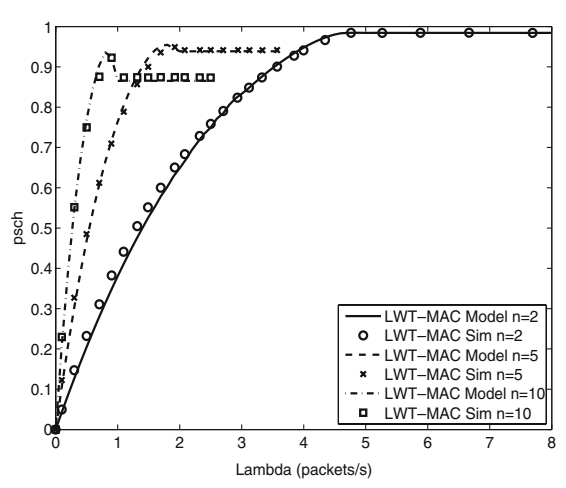

(a) Scheduled Probability

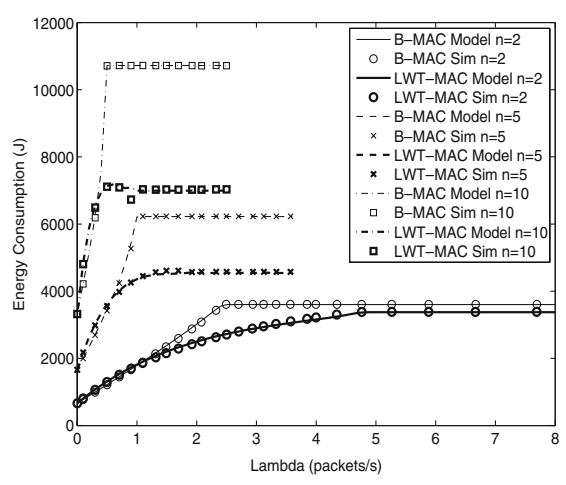

(b) Energy Consumption

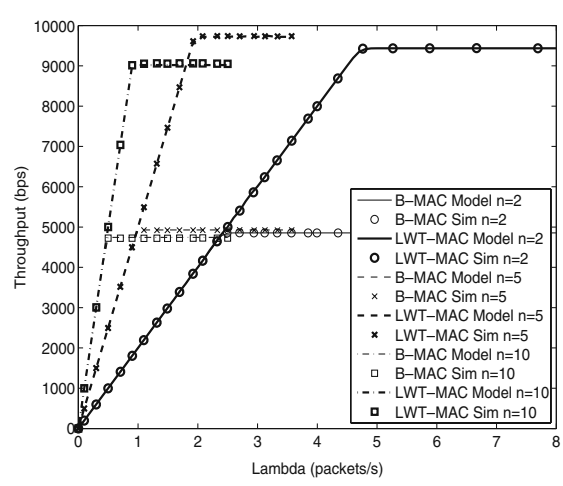

(c) Throughput

Fig. 5 Scheduled probability, total energy consumption and throughput with different number of sensor nodes

put decreases with ten nodes as the collision probability increases.

Figure 6 shows the results with ten nodes and different check intervals (the $T_{\text {listen }}$ value is maintained while $T_{\text {sleep }}$ changes to fit the given check interval). The scheduled probability of LWT-MAC (Fig. 6a) remains equal for all the check intervals at high loads, however at lower loads it increases faster with higher values of the check interval. This difference occurs because with longer check intervals the probability that a node has received something to transmit during an ongoing transmission increases. In the energy consumption (Fig. 6b) it can be seen, for both protocols, that at low loads the use of short time intervals notably penalizes the energy consumption as nodes wake up unnecessarily more often. However, as the load increases, to have longer check intervals in LWT-MAC implies higher energy consumption caused by the collisions that move the network to the unscheduled mode (moreover, after a collision nodes wait awake listening the entire preamble transmission). However, the obtained energy consumption values at high loads are substantially lower than the ones obtained using B-MAC. In the through- put (Fig. 6c), it can be observed that lower saturation values are obtained when longer preambles are used, in both cases: using B-MAC and LWT-MAC. In this case, once again, it can be seen how the LWT-MAC improves the B-MAC performance increasing notably the saturation throughput.

\section{Optimization analysis}

In this section, a performance optimization is done in order to derive the best parameter configuration of the LWT-MAC protocol for a single-hop scenario. A first goal is to identify how the different parameters that define the LWT-MAC operation affect its performance and how they can be tuned to minimize the energy consumption without significantly reducing the throughput and delay.

\subsection{Key parameters optimization}

One of the parameters of crucial importance is the sleep time of the duty cycle ( $\left.T_{\text {sleep }}\right)$ as it directly affects

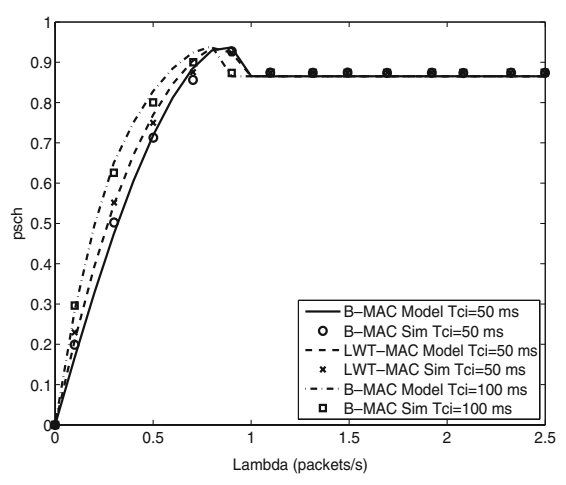

(a) Scheduled Probability

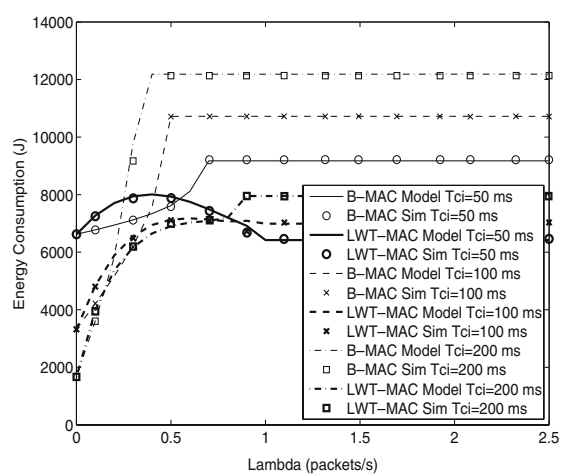

(b) Energy Consumption

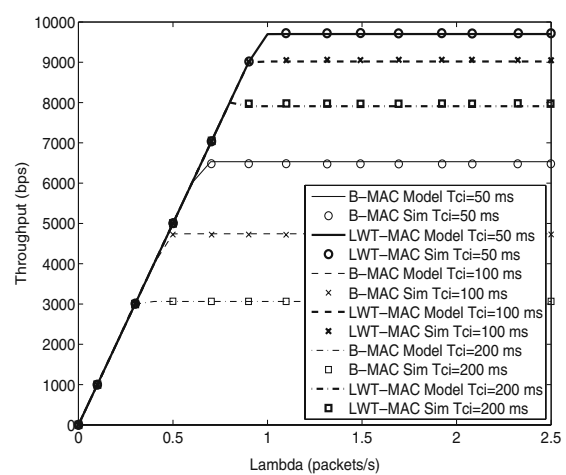

(c) Throughput

Fig. 6 Scheduled probability, total energy consumption and throughput with different values of the check interval 
the performance of the network. High values of the $T_{\text {sleep }}$ allow to decrease the energy consumption but at the cost of reducing the throughput and increasing the delay. Additionally, it is possible to define a probability of waking up after successful transmissions $\left(p_{\mathrm{w}}\right)$, based on which sensor nodes decide to wake up at the end of a successful transmission or go to sleep remaining in the unscheduled access. Observe that, an additional mechanism should be implemented to inform nodes to wake up at the end of each transmission based on this probability, otherwise receivers can be sleeping when nodes send packets without using the long preamble. The sender can, for instance, notify sensor nodes to wake up at the end of the transmission in the data or RTS messages. At low traffic loads, setting that probability to small values allows to reduce the energy consumption as fewer idle listening after transmission periods will occur, however, as load increases, higher values of $p_{\mathrm{w}}$ reduce the energy waste since the number of long preamble transmissions are reduced. Note that by defining this probability Eqs. 13, 18, 21 and 26 should be rewritten as shown in Eqs. 29, 30, 31 and 32, respectively:

$p_{\text {sch }}=p_{\mathrm{w}} \frac{p_{\text {ss }}}{1-p_{\text {es }}}\left(1-(1-\rho)^{n}\right), \quad p_{\text {unsch }}=1-p_{\text {sch }}$

$$
\begin{aligned}
e_{\mathrm{s}, \mathrm{tx}}= & E_{\mathrm{idle}}\left(\mathrm{DIFS}+B \sigma p_{\mathrm{e}}+3 \operatorname{SIFS}+p_{\mathrm{w}} T_{\mathrm{e}}\right) \\
& +E_{\mathrm{tx}} \frac{\left(L_{\mathrm{p}} \cdot p_{\mathrm{unsch}}\right)+L_{\mathrm{rts}}+L_{\mathrm{data}}}{r} \\
& +E_{\mathrm{rx}} \frac{L_{\mathrm{cts}}+L_{\mathrm{ack}}}{r}
\end{aligned}
$$

$$
\begin{aligned}
e_{\mathrm{s}, \mathrm{rx}}= & p_{\mathrm{sch}} \cdot e_{\mathrm{b}}+p_{\text {unsch }} \cdot e_{\mathrm{p}}+E_{\mathrm{idle}}\left(3 \operatorname{SIFS}+p_{\mathrm{w}} T_{\mathrm{e}}\right) \\
& +E_{\mathrm{rx}}\left(\frac{L_{\mathrm{rts}}+L_{\mathrm{data}}}{r}\right)+E_{\mathrm{tx}}\left(\frac{L_{\mathrm{cts}}+L_{\mathrm{ack}}}{r}\right)
\end{aligned}
$$

$$
\begin{aligned}
e_{\mathrm{s}, \mathrm{ov}}= & p_{\mathrm{sch}} \cdot e_{\mathrm{b}}+p_{\mathrm{unsch}} \cdot e_{\mathrm{p}}+E_{\mathrm{idle}} p_{\mathrm{w}} T_{\mathrm{e}}+E_{\mathrm{rx}} \frac{L_{\mathrm{rts}}}{r} \\
& +E_{\text {sleep }}\left(\frac{L_{\mathrm{cts}}+L_{\mathrm{data}}+L_{\mathrm{ack}}}{r}+3 \mathrm{SIFS}\right)
\end{aligned}
$$

Other parameters such as the $\mathrm{CW}$, used to compute the random $\mathrm{BO}$, also affect the performance, but compared to the $T_{\text {sleep }}$ and $p_{\mathrm{w}}$ their influence is limited. Parameters like the packet length or the traffic load are considered fixed.

\subsection{Optimization function}

The main goal of the optimization process is to minimize the energy consumption (e) but constrained to achieve the same throughput as the IEEE 802.11 (CSMA/CA) MAC protocol. The IEEE 802.11 has been chosen as a reference as it does not implement the duty cycle operation, resulting in an upper bound in terms of performance (given that the other common parameters, such as the CW and the RTS/CTS option are equally configured in both approaches). The optimization analysis considers the throughput as the only constraint since the average packet transmission delay will necessarily increase to allocate space for the long preamble transmission, which is the price that the LWT-MAC pays to obtain a lower energy consumption than the IEEE 802.11 without reducing its throughput. The optimization function is shown in Eq. 33.

$$
\begin{aligned}
& {\left[T_{\text {sleep }}^{*}, p_{\mathrm{w}}^{*}\right]} \\
& \quad=\underset{T_{\text {sleep }} \in[0,0.5], p_{\mathrm{w}} \in[0,1], S \geq S_{802.11}}{\arg \min } e\left(n, L_{\text {data }}, \zeta, T, T_{\text {sleep }}, p_{\mathrm{w}}\right)
\end{aligned}
$$

Observe that, the metrics referred by $\zeta$ (see Table 2) are a function of $n, \lambda$ and $L_{\text {data }}$ and can be obtained using the previously described analytical model.

\subsection{Optimization results}

By applying Eq. 33 the optimal sleep time $\left(T_{\text {sleep }}^{*}\right)$ and wake up probability $\left(p_{w}^{*}\right)$ can be obtained for a given scenario. The optimal values are those that minimize the energy consumption and achieve the same throughput as the IEEE 802.11. To see how the optimal parameters change for different traffic loads $(A)$ and number of nodes $(n)$, a single-hop network with the parameters shown in Table 1 (see Section 3) has been evaluated.

Results are shown in Fig. 7. Note that, once the throughput of IEEE 802.11 cannot be achieved the metrics are not longer depicted. The minimum energy consumption (Fig. 7b) for the achieved throughput is depicted in Fig. 7a. Observe, in Fig. 7c, how the optimal probability to wake up after a successful transmission $\left(p_{\mathrm{w}}^{*}\right)$ increases rapidly from 0 to 1 when the traffic load starts to be noticeable. In contrast, the $T_{\text {sleep }}^{*}$ (Fig. 7f) takes high values at low loads, meaning that, for the load requirements, sensor nodes can be sleeping during a longer time in each duty cycle. As the load increases, the value of $T_{\text {sleep }}^{*}$ decreases, however it shows an inflection point and begins to increase. This effect is caused by the listening after transmissions probability that suddenly increases to 1 reducing the offered load of the network as it reduces the time needed to send a message. After this behavior, the $T_{\text {sleep }}^{*}$ continues decreasing in order to maintain the throughput but also 


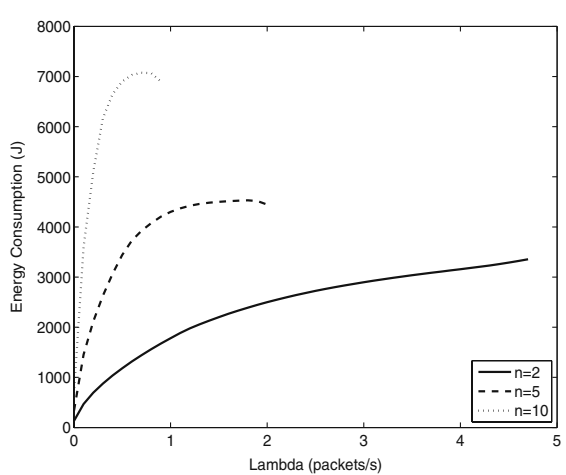

(a) Energy Consumption

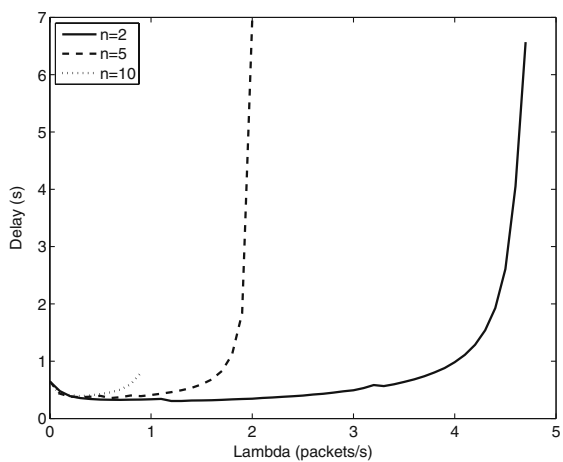

(d) Delay

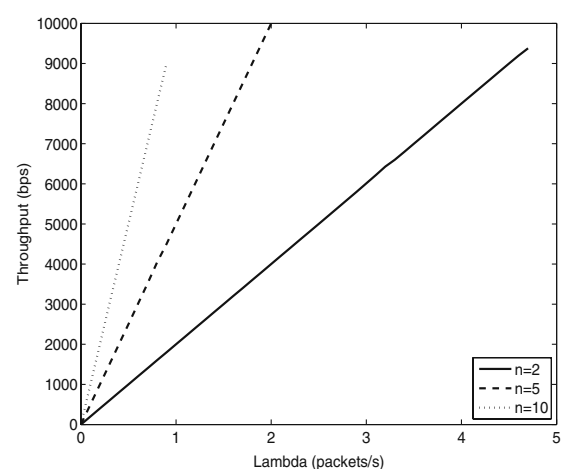

(b) Throughput

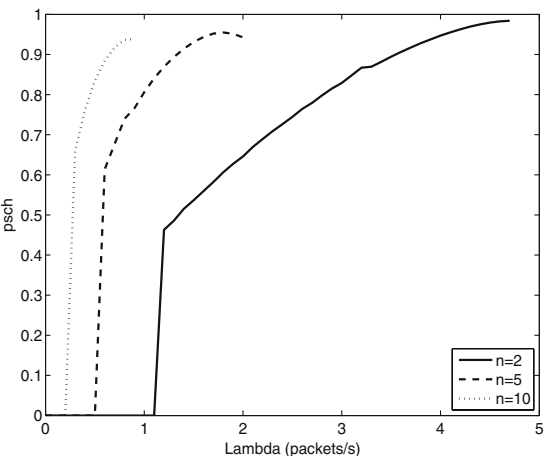

(e) Scheduled Probability

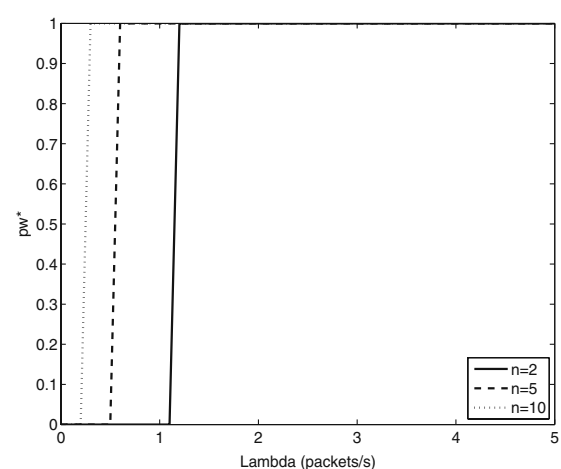

(c) $p_{\mathrm{w}}^{*}$

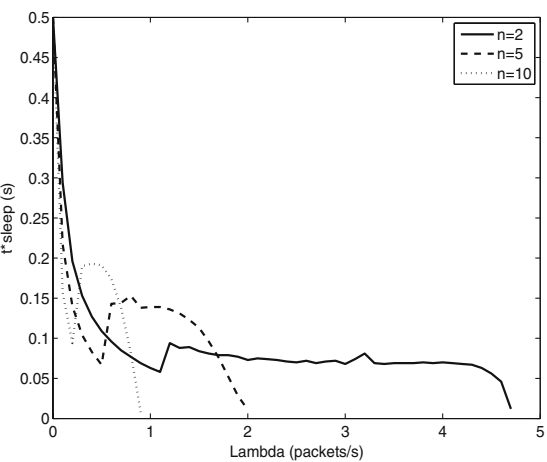

(f) $T_{\text {sleep }}^{*}$

Fig. 7 Performance metrics with optimum $T_{\text {sleep }}^{*}$ and $p_{\mathrm{w}}^{*}$ in a single-hop network

to reduce the duration of collisions. Moreover, as it has been considered that after a collision nodes also keep listening to the channel, thus receiving the entire next long preamble transmission, the consumption with high traffic loads increases with the value of the sleep time (that makes the long preamble to increase). The values $T_{\text {sleep }}^{*}$ and $p_{\mathrm{w}}^{*}$ directly influence the delay and the scheduled probability. The delay, depicted in Fig. $7 d$, shows a small increase at very low loads caused by the long sleep time and remains more or less constant until the queues become saturated. Regarding the scheduled probability (Fig. 7e), it is directly affected by $p_{\mathrm{w}}^{*}$ that bounds its value at low loads.

From these results, it can be concluded that, if the load can be estimated, the best configuration is: $a$ ) at low loads set the $T_{\text {sleep }}$ to long values and $p_{\mathrm{w}}$ equal 0 and $b$ ) at high loads decrease the $T_{\text {sleep }}$ value and set $p_{\mathrm{w}}$ equal 1 .

\subsection{Heuristic configuration of the LWT-MAC parameters}

The estimation of the traffic load is a difficult task, even more in event-based WSNs where the traffic profiles differ from the traditional ones, showing sporadic and instantaneous increases of the traffic load due, for instance, to events occurrence. Moreover, sensor nodes are devices with limited capabilities in terms of processing and memory resources making the load estimation an arduous task. However, if the load can be estimated in a fast and reliable way, the best option will be to use the LWT-MAC with the optimal values for $T_{\text {sleep }}$ and $p_{\mathrm{w}}$. Otherwise, from the observations made in the optimization analysis performed in the previous section, a heuristic parameter configuration to provide low energy consumption, high throughput and small delay through the entire load range can be made.

The major disadvantage of LWT-MAC is that it consumes more energy than B-MAC at low loads due to idle listening after transmission periods, however a moderately long value of $T_{\text {sleep }}$ can help to decrease the energy consumption at low loads. To benefit from the advantages of LWT-MAC, if the $T_{\text {sleep }}$ is fixed to a long value, the $p_{\mathrm{w}}$ probability should always be set to one. With this configuration, the LWT-MAC will consume less energy at low loads and it will maintain its capability to react to instantaneous increases of the network load.

Figure 8 shows for different number of sensor nodes the value of the energy consumption and throughput 


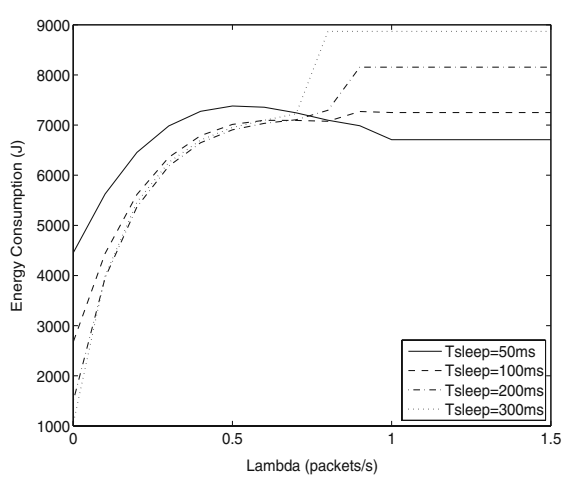

(a) Energy Consumption $n=10$

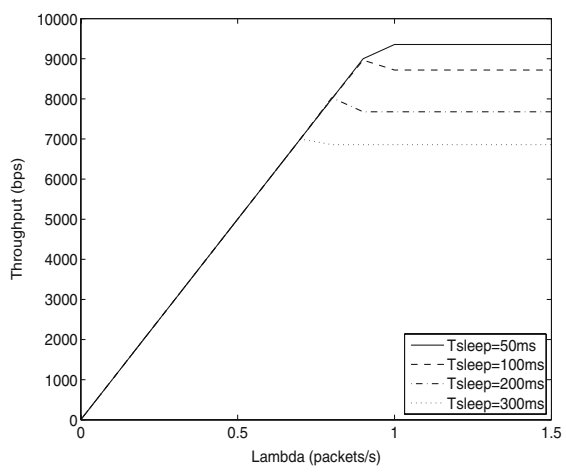

(d) Throughput $n=10$

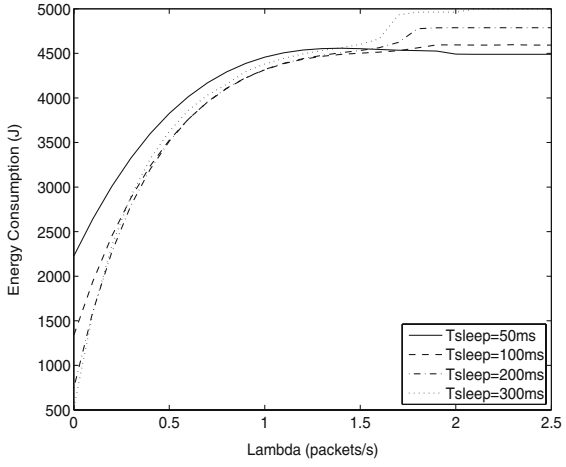

(b) Energy Consumption $n=5$

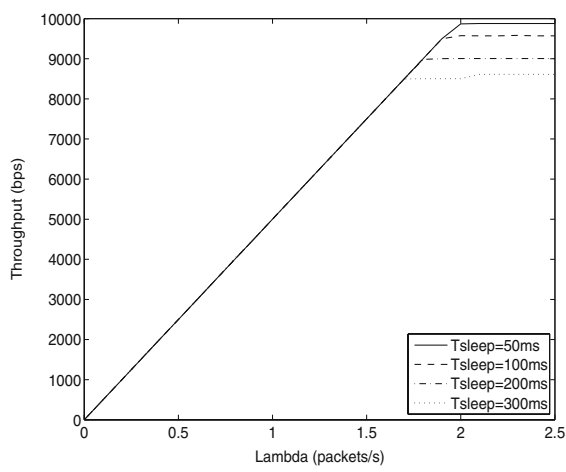

(e) Throughput $n=5$

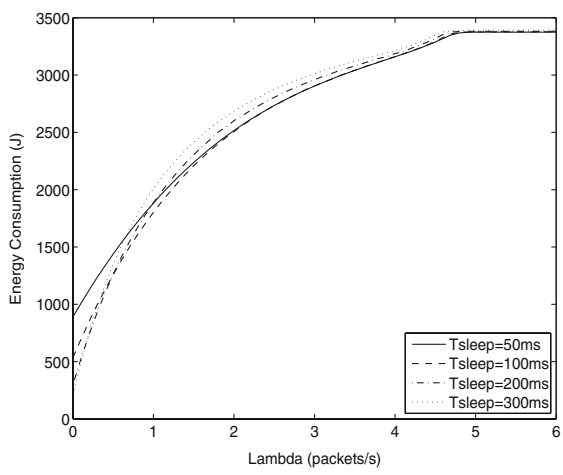

(c) Energy Consumption $n=2$

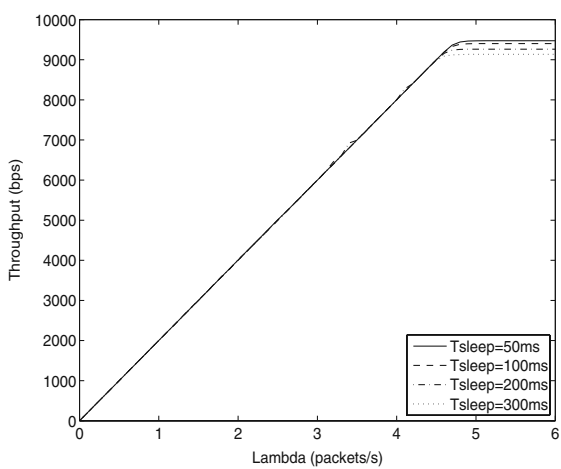

(f) Throughput $n=2$

Fig. 8 Performance metrics for different $T_{\text {sleep }}$ and $p_{\mathrm{w}}=1$ in a single-hop network

with different values of $T_{\text {sleep }}$ and varying the traffic load. It can be seen that for $T_{\text {sleep }}=50 \mathrm{~ms}$ the energy consumption at low loads is significantly higher. However, it decreases substantially with $T_{\text {sleep }}=100$ $\mathrm{ms}$ and, still a bit more, with $T_{\text {sleep }}=200 \mathrm{~ms}$. However, with $T_{\text {sleep }}=300 \mathrm{~ms}$ the reduction of energy consumption at low loads is extremely small, compared to the obtained with $200 \mathrm{~ms}$, and at the cost of a lower throughput. Therefore, a $T_{\text {sleep }}$ around $200 \mathrm{~ms}$ provides a considerably reduction of the energy consumption at low loads maintaining an acceptable value for the throughput. However, once the network saturates the energy consumption increases with higher values of $T_{\text {sleep }}$ since it is considered that all nodes keep listening to the channel after a collision, thus receiving the entire long preamble of the retransmissions. Nevertheless, in the normal operation, the network is expected to work from low to moderate load conditions, not in saturation.

Thus, the suggested heuristic configuration is: $T_{\text {sleep }}=200 \mathrm{~ms}$ and $p_{\mathrm{w}}=1$.

\section{Performance evaluation}

In this section, the heuristic configuration of $T_{\text {sleep }}=$ $200 \mathrm{~ms}$ and $p_{\mathrm{w}}=1$ is evaluated and compared to B-
MAC with $T_{\text {sleep }}=75 \mathrm{~ms}$ in a single-hop and a multihop network. The results of LWT-MAC with $T_{\text {sleep }}=$ $75 \mathrm{~ms}$ and $p_{\mathrm{w}}=1$ have also been included to keep them

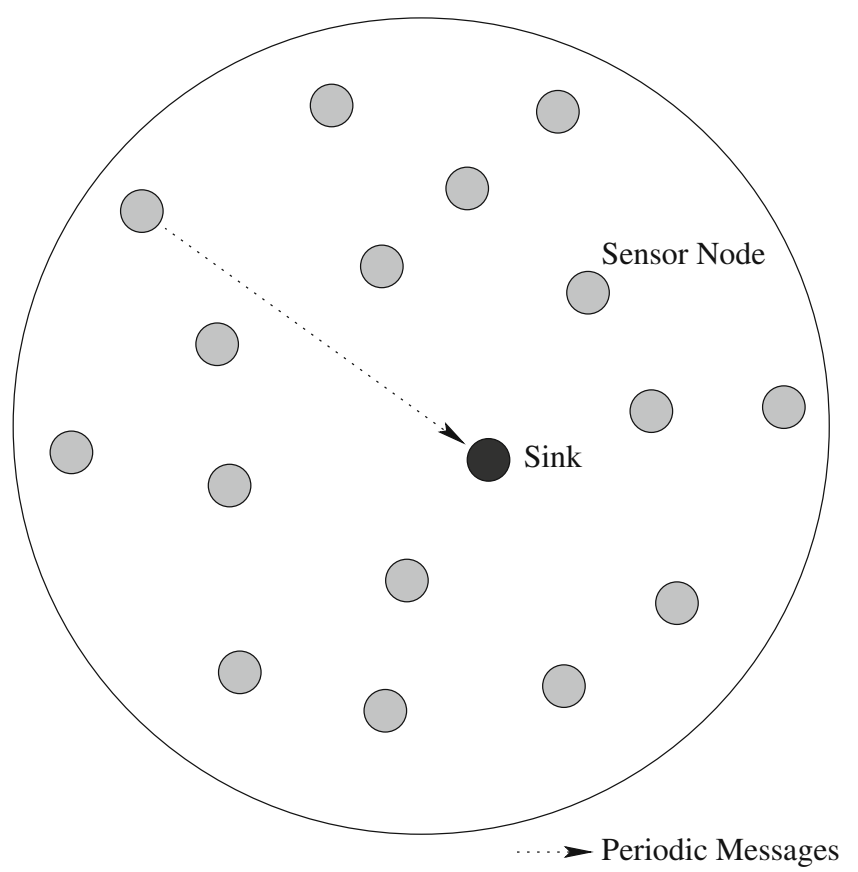

Fig. 9 Single-hop scenario 


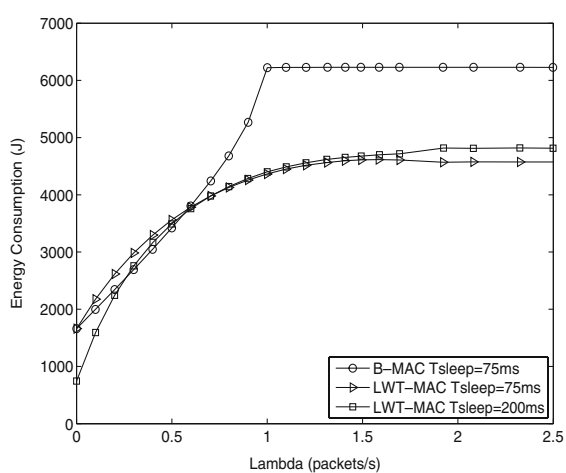

(a) Energy Consumption $n=5$

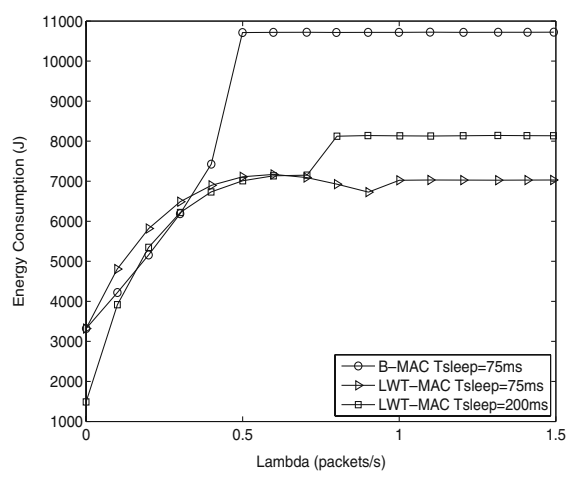

(d) Energy Consumption $n=10$

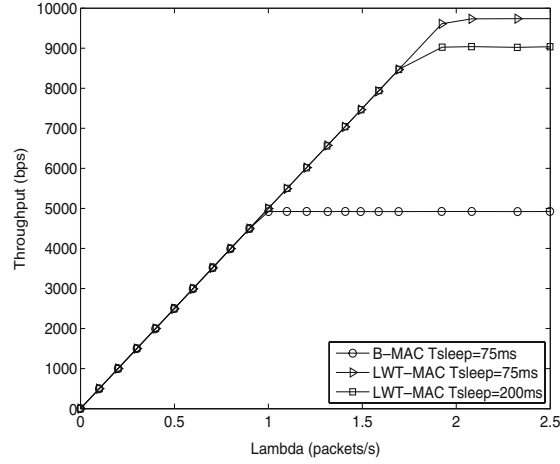

(b) Throughput $n=5$

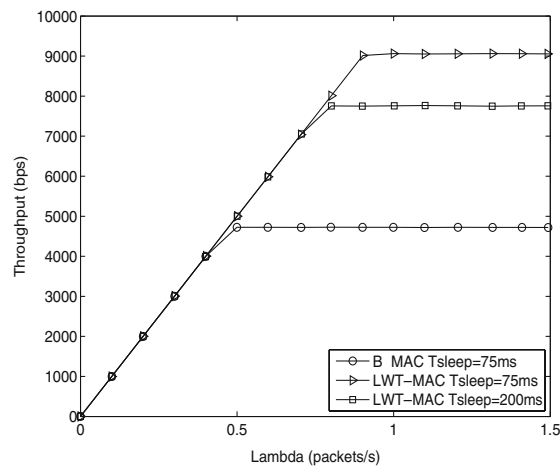

(e) Throughput $n=10$

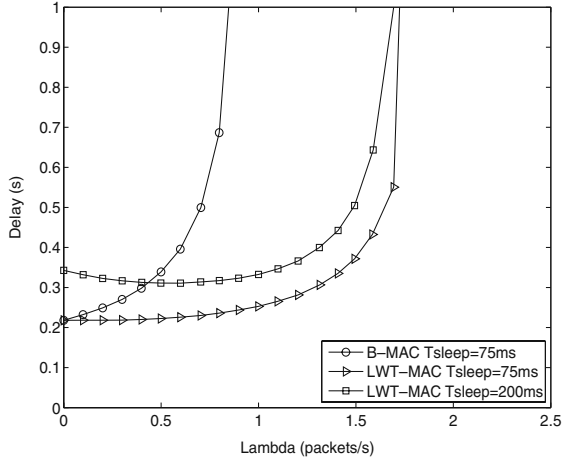

(c) Delay $n=5$

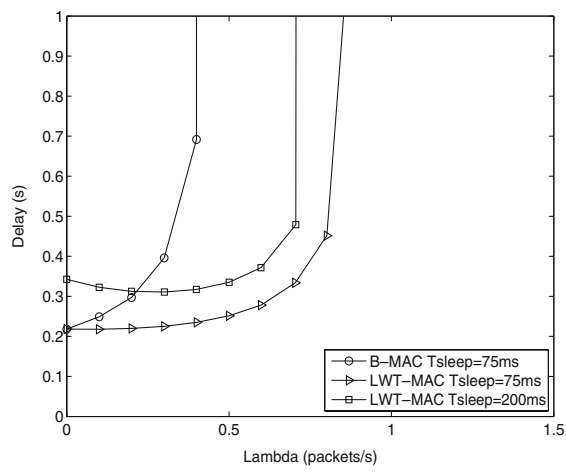

(f) Delay $n=10$

Fig. 10 Performance metrics with recommended heuristic $T_{\text {sleep }}$ and $p_{\mathrm{w}}$ in a single-hop network with five and ten sensor nodes

as a reference. For a fair comparison the RTS/CTS procedure is used in both B-MAC and LWT-MAC (for the scheduled and unscheduled accesses), therefore an RTS is sent immediately after the long preamble transmission. After overhearing an RTS or CTS message, nodes go to sleep for the ongoing transmission duration in both B-MAC and LWT-MAC. In the multi-hop case event-based traffic profiles will be also considered.

The SENSE simulator [21], as explained before, has been used to obtain the results. In this case the simulator has also been extended with the B-MAC protocol and the event-based traffic profile. The channel has been considered error-free.

\subsection{Single-hop network}

The single-hop scenario consists of $n$ nodes randomly placed in an area smaller than the maximum coverage range. All sensor nodes generate messages following a Poisson distribution and send them to the sink (Fig. 9). The default parameters used for the evaluation are shown in Table 1 (see Section 3). In this scenario the CA timer is deactivated.

Observe that this scenario provides the best conditions for the LWT-MAC protocol since without hid- den terminals and channel errors all sensor nodes will synchronize to ongoing transmissions and therefore, will be able to send their packets after them without making use of the long preamble. Moreover, since all the nodes are inside the coverage range of the others, receivers are always awake in the scheduled phase, i.e., they overhear all transmissions taking place. The only

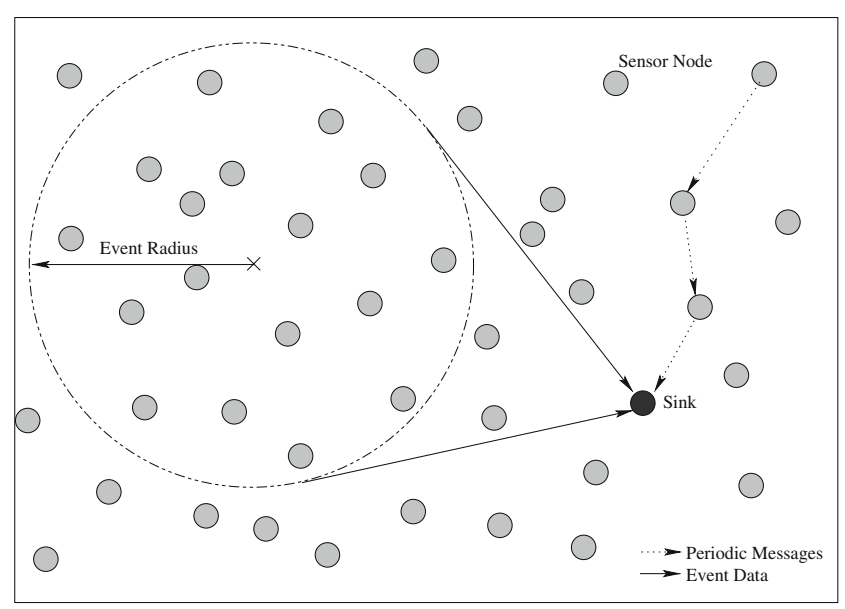

Fig. 11 Multi-hop scenario with periodic and event-based traffic profiles 
Table 3 Default parameters

\begin{tabular}{llll}
\hline Parameter & Value & Parameter & Value \\
\hline$r$ (data rate) & $20 \mathrm{kbps}$ & $T_{\text {listen }}$ & $24.5 \mathrm{~ms}$ \\
$\sigma$ (empty slot) & $1 \mathrm{~ms}$ & $L_{\text {data }}($ packet size $)$ & $240 \mathrm{bits}$ \\
DIFS & $10 \mathrm{~ms}$ & $L_{\mathrm{rts}}, L_{\text {cts }}, L_{\text {ack }}$ & $64 \mathrm{bits}$ \\
SIFS & $5 \mathrm{~ms}$ & $E_{\mathrm{tx}}$ & $24.75 \mathrm{~mW}$ \\
$\mathrm{CW}$ & 64 & $E_{\mathrm{rx}}, E_{\text {idle }}$ & $13.5 \mathrm{~mW}$ \\
$K$ (queue size) & $10 \mathrm{pkts}$ & $E_{\text {sleep }}$ & $0.015 \mathrm{~mW}$ \\
$R$ (retry limit) & 5 & $T$ (time) & $5 \cdot 10^{5} \mathrm{~s}$ \\
\hline
\end{tabular}

limitation of this scenario is the occurrence of collisions. It has been assumed that collisions move the system to the unscheduled phase in which nodes should use the long preamble before a data transmission. However, after the initial transmission in the unscheduled phase the system will immediately move to the scheduled phase again.

Results (Fig. 10) show that the energy consumption of LWT-MAC with the heuristic parameter configuration is similar to the one obtained by B-MAC at low loads as can be observed in Fig. 10a and d. However, at high loads the suppression of the long preamble reduces the energy consumption of the LWTMAC protocol. The suppression of the long preamble transmission is the cause of the higher throughput of the LWT-MAC compared to the B-MAC as depicted in Fig. 10b and e. However, the increase of the $T_{\text {sleep }}$ to maintain the energy consumption makes the delay to slightly increase at low loads as can be seen in Fig. 10c and $\mathrm{f}$.

Compared to the LWT-MAC with $T_{\text {sleep }}=75 \mathrm{~ms}$, the heuristic configuration provides slightly worse results in terms of throughput and delay but considerably reduces the energy consumption at low loads.

\subsection{Multi-hop network}

The multi-hop scenario consists of a multi-hop eventbased WSN with 100 nodes randomly placed in a $100 \times$ $100 \mathrm{~m}^{2}$ area. The radio range of each node is $43 \mathrm{~m}$ and the Floyd algorithm has been used to compute the shortest path between any pair of nodes. Each sensor node generates two kinds of traffic profiles: (i) messages generated following a Poisson distribution and (ii) event-based messages (see Fig. 11). A random event generator selects randomly the event position and notifies the sensor nodes that are inside the coverage radius of the event in order to send event-based messages

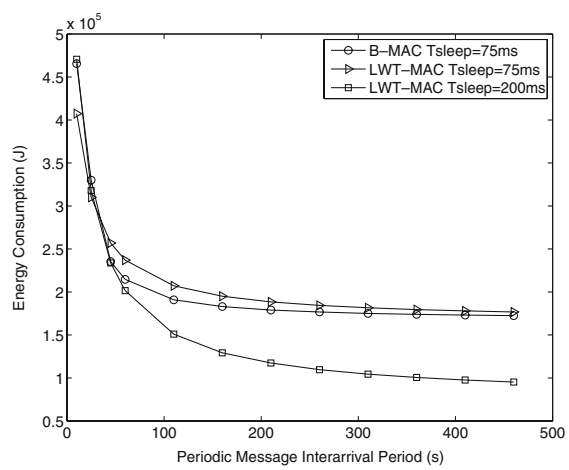

(a) Energy Consumption

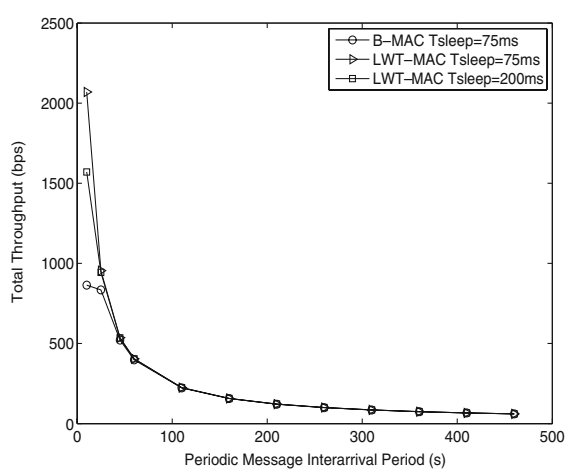

(b) Throughput

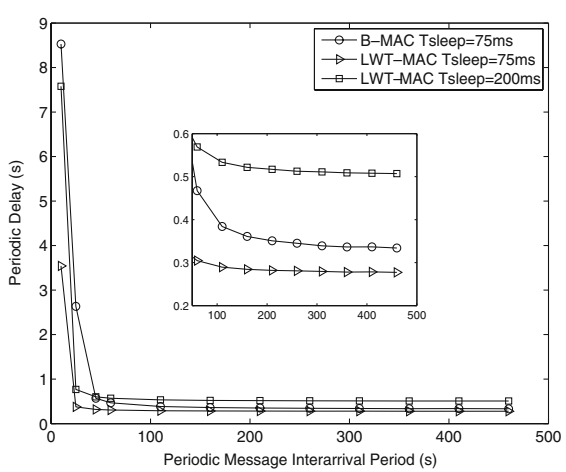

(c) Delay of periodic messages

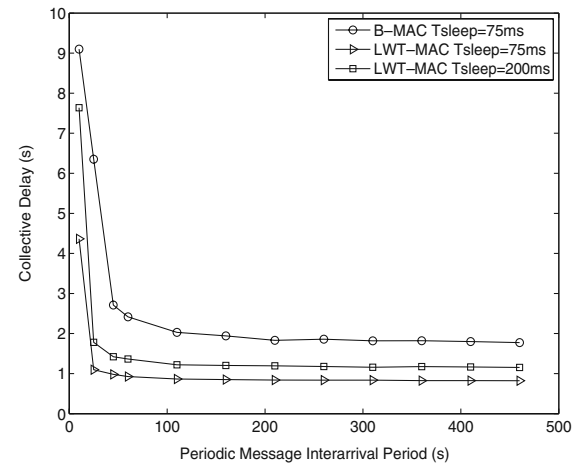

(d) Collective Delay

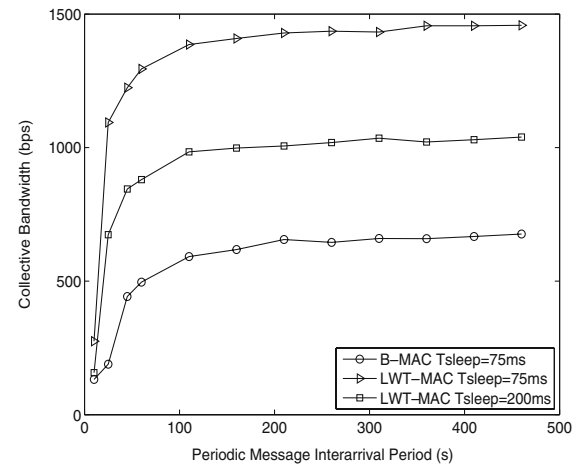

(e) Collective Bandwidth

Fig. 12 Performance metrics with recommended heuristic $T_{\text {sleep }}$ and $p_{\mathrm{w}}$ in a multi-hop network with periodic and event-based traffic profiles 
Table 4 Collective reliability for periodic interarrival time equals 10 and $25 \mathrm{~s}$

\begin{tabular}{lll}
\hline MAC protocol & $10 \mathrm{~s}$ & $25 \mathrm{~s}$ \\
\hline B-MAC $T_{\text {sleep }}=75 \mathrm{~ms}$ & 0.509 & 0.995 \\
LWT-MAC $T_{\text {sleep }}=75 \mathrm{~ms}$ & 0.992 & 0.999 \\
LWT-MAC $T_{\text {sleep }}=200 \mathrm{~ms}$ & 0.891 & 0.999 \\
\hline
\end{tabular}

to the sink. Events have a constant coverage radius of $30 \mathrm{~m}$, the time between events follows an exponential distribution with mean $600 \mathrm{~s}$ and the number of event messages needed at sink to reliably detect an event has been set to 5 . In this scenario the CA timer is activated. The parameters used are shown in Table 3.

The results obtained are shown in Fig. 12. It is observed that at low loads the energy consumption of the LWT-MAC with the heuristic configuration is considerably lower than using the B-MAC (Fig. 12a). This effect appears due to the longer $T_{\text {sleep }}$ but also due to the CA timer that avoids continuous collisions of preambles and better manages hidden terminal problems. The LWT-MAC provides also better results in throughput, depicted in Fig. 12b, with a higher value of the saturation throughput and delay (Fig. 12c).

The collective QoS metrics are shown in Fig. 12d, e and Table 4. The LWT-MAC with heuristic configuration achieves lower collective delay (Fig. 12d), defined as the time span between the event occurrence and the event detection at sink [22]. It also provides better collective bandwidth for the event-based messages as shown in Fig. 12e and better collective reliability (see Table 4) for a message interarrival period equals 10 and $25 \mathrm{~s}$. For other loads the reliability is almost $100 \%$ in all the cases. Collective bandwidth refers to the bandwidth required to detect an event while collective reliability is the fraction of correctly detected events among all events generated [18].

Observe that, the LWT-MAC with $T_{\text {sleep }}=75 \mathrm{~ms}$ provides, as seen in the single-hop scenario, better results in throughput and delay but also in the collective metrics. However, the heuristic configuration allows to noticeably decrease the energy waste at low loads by obtaining better collective and individual metrics than B-MAC.

\section{Concluding remarks}

In this work an analysis of the LWT-MAC protocol has been performed. A LWT-MAC analytical model that computes the network performance metrics and the energy consumption taking into account collisions in both saturated and unsaturated conditions has been presented. Moreover, the optimal configuration for the probability to wake up after a successful transmission $\left(p_{\mathrm{w}}\right)$ and the sleep time of the duty cycle $\left(T_{\text {sleep }}\right)$ have been obtained depending on the number of nodes and the load of the network in a single-hop scenario. From the optimization results, a heuristic configuration for the $T_{\text {sleep }}$ and the $p_{\mathrm{w}}$ is suggested. The use of the proposed heuristic parameter configuration avoids the complexity of other mechanisms that, for example, adapt the parameters based on the traffic load estimation, which can be unfeasible in WSNs, and provides a near-optimal performance in a wide range of situations. Additionally, the LWT-MAC with the heuristic parameter configuration has been compared with BMAC in a single-hop network as well as in a multi-hop scenario with event-based traffic. Results show that the energy consumption of the sensor nodes is maintained similar or lower to the consumed by B-MAC and that the other performance metrics, specially those regarding to collective QoS, are substantially improved. Although the heuristic parameter configuration has been obtained for a single-hop scenario, it has been shown that it is also valid in a multi-hop network.

Acknowledgements This work has been partially supported by the Spanish Government under the projects TEC2008-0655/TEC (GEPETO, Plan Nacional I+D) and CSD2008-00010 (COMONSENS, Consolider-Ingenio Program) and by the Catalan Government (SGR2009\#00617)

\section{References}

1. 802.11, IS (1999) Wireless LAN medium access control (MAC) and physical layer (PHY) specifications. ANSI/IEEE Std 802.11. Revised 2007

2. Polastre J, Hill J, Culler D (2004) Versatile low power media access for wireless sensor networks. In: Proceedings of the 2nd international conference on embedded networked sensor systems (SenSys '04)

3. Cano C, Bellalta B, Sfairopoulou A, Barceló J (2009) A low power listening MAC with scheduled wake up after transmissions for WSNs. IEEE Commun Lett 13(4):221-223

4. Ye W, Heidemann J, Estrin D (2004) Medium access control with coordinated adaptive sleeping for wireless sensor networks. IEEE/ACM Trans Netw 12(3):493-506

5. Haapola J (2005) Multihop medium access control for WSNs: an energy analysis model. EURASIP J Wirel Comm 2005(4):523-540

6. Zhang Y, He C, Jiang L (2008) Performance analysis of SMAC protocol under unsaturated conditions. IEEE Commun Lett 12(3):210-212

7. Buettner M, Yee G, Anderson E, Han R (2006) X-MAC: a short preamble MAC protocol for duty-cycled wireless sensor networks. In: Proceedings of the 4th international conference on embedded networked sensor systems (Sensys '06), pp 307320

8. Ye W, Silva F, Heidemann J (2006) Ultra-low duty cycle MAC with scheduled channel polling. In: Proceedings of the 
4th international conference on embedded networked sensor systems, p 334

9. El-Hoiydi A, Decotignie J, Hernandez J (2004) Low power MAC protocols for infrastructure wireless sensor networks. In: Proceedings of the fifth European wireless conference, pp $563-569$

10. van Dam T, Langendoen K (2003) An adaptive energyefficient MAC protocol for wireless sensor networks. In: Proceedings of the first international conference on embedded networked sensor systems (SenSys '03), p 171. http://portal. acm.org/citation.cfm?doid=958491.958512

11. Chen D, Varshney P (2004) QoS support in wireless sensor networks: a survey. In: Proceedings of the international conference on wireless networks (ICWN'04), pp 227-233

12. Paek K, Kim J, Song U, Hwang C (2007) Priority-based medium access control protocol for providing QoS in wireless sensor networks. IEICE Trans Inf Sys 90(9):1448

13. Liu Y, Elhanany I, Qi H (2005) An energy-efficient QoSaware media access control protocol for wireless sensor networks. In: Proceedings of the international conference on mobile adhoc and sensor systems conference, pp 189 191

14. Liu Z, Elhanany I (2006) RL-MAC: a QoS-aware reinforcement learning based MAC protocol for wireless sensor networks. In: Proceedings of the IEEE international conference on networking, sensing and control (ICNSC'06), pp 768773

15. Caccamo M, Zhang L, Sha L, Buttazzo G (2002) An implicit prioritized access protocol for wireless sensor networks. In:
Proceedings of the 23rd IEEE real-time systems symposium (RTSS'02)

16. Watteyne T, Augé-Blum I, Ubéda S (2006) Dual-mode real-time MAC protocol for wireless sensor networks: a validation/simulation approach. In: Proceedings of the 1st international conference on integrated internet ad hoc and sensor networks

17. Lu G, Krishnamachari B, Raghavendra C (2004) An adaptive energy-efficient and low-latency MAC for data gathering in wireless sensor networks. In: Proceedings of the parallel and distributed processing symposium

18. Cano C, Bellalta B, Barceló J, Sfairopoulou A (2009) A novel MAC protocol for event-based wireless sensor networks: improving the collective QoS. In: Proceedings of the 7th international conference on wired / wireless internet communications (WWIC)

19. Alizadeh-Shabdiz F, Subramaniam S (2006) Analytical models for single-hop and multi-hop ad hoc networks. Mob Netw Appl 11(1):75-90

20. Bellalta B, Oliver M, Meo M, Guerrero M (2005) A simple model of the IEEE 802.11 MAC protocol with heterogeneous traffic flows. In: Proceedings of the IEEE Eurocon

21. Chen G, Branch J, Pflug M, Zhu L, Szymanski B (2004) SENSE: a sensor network simulator. In: Advances in pervasive computing and networking, pp 249-69

22. Lin X, Zhou J, Mu C (2006) Collective real-time QoS in wireless sensor networks. In: Proceedings of the international conference on wireless communications, networking and mobile computing (WiCOM'06), pp 1-4 